\title{
Strain Transfer Analysis of Embedded Fiber Bragg Grating Strain
}

\section{Sensor}

\author{
Li Sun ${ }^{a}$, Hong Hao ${ }^{b}$, Bo Zhang ${ }^{a}$, Xueling Ren ${ }^{a}$, Jun Li ${ }^{b}$ \\ a School of Civil Engineering, Shenyang Jianzhu University, \#9 Hunnan East Road, Shenyang, China, 110168 \\ b Centre for Infrastructural Monitoring and Protection, School of Civil and Mechanical Engineering, Curtin \\ University, Kent Street, Bentley, WA 6102, Australia
}

\begin{abstract}
Due to the difference in the physical and mechanical properties between the optical fiber, protective layer, adhesive layer and the host material, the strains measured by a Fiber Bragg Grating (FBG) sensor may not be the actual strains of the host material, which impedes the reliable applications of FBG sensors. To overcome this problem, in this paper, the strain transfer formula is derived by using elastic analysis, shear-lag method and several reasonable assumptions taking into account the temperature variations and non-axial stresses, which is an improvement to an existing study ( $\mathrm{Li}$ et al. 2007). The analytical results are validated by simulating the behavior of a bare optical fiber through finite element analysis. The data have enabled identifying the parameters that influence the strain transfer from the host material to the embedded FBG sensors, as well as the impact of the temperature variation and sensor alignment angle on the measurement accuracy. This work provides additional knowledge for the improvement of the existing strain transfer theory of FBG sensors in order to achieve a more accurate strain measurement with this sensing technology.
\end{abstract}

KEYWORDS: FBG sensor, strain transfer, finite element analysis, non-axial stress, temperature

Address all correspondence to: Li Sun, School of Civil Engineering, Shenyang Jianzhu University, \#9 Hunnan East Road, Shenyang, China, 110168; Tel:+86 24-24694309; Fax:+86 24-24691900; Email: sunli2000sy@163.com

\section{Introduction}

Strain is an important indicator of the engineering structure's condition, and therefore it is usually measured and used for the assessment of structural service conditions and safety. If local strain in a structure subjected to various loadings is too large, structural damage, such as cracks could be induced, and the growth and propagation of cracks may threaten the safety and service life of the structure. Since Fiber Bragg Grating (FBG) sensors have a number of significant advantages, such as small size, high sensitivity, anti-corrosion ability, immunity to 
electromagnetic interferences, capability of distributed or quasi-distributed measurement, and suitability for long-distance monitoring and data transmission, the sensing technology based on FBG sensors has become a favorable solution to measure the strains for structural health monitoring in civil engineering.

Numerous scholars have investigated the strain transfer mechanism of FBG sensors. Cox [1] first proposed the application of shear-lag theory to study the load transfer between constituent materials in paper and other fibrous materials, and provided a theoretical basis for the research of strain transfer between the embedding material and the optical fiber of FBG sensors. Nanni et al. [2] presented a nondestructive testing method based on fiber-optics for in situ measurements of strain-stress in concrete structures, and the two most promising techniques for concrete structure diagnostics were demonstrated in the laboratory using concrete cylinders subjected to unconfined uniaxial compression with optical fibers embedded in the direction parallel and perpendicular to the applied strain (load). Pak [3] analyzed the influence of the coating layer's modulus of elasticity and thickness on the strain transfer to a coated optical fiber, as well as the stress and strain concentrations caused by them. The study considered the case of a far-field longitudinal shear stress parallel to both the optical fiber and structural fibers, and the host structure as an infinite, isotropic and homogeneous material. Ansari et al. [4] reported a mechanical model to estimate the actual level of structural strains from the values measured by an optical fiber sensor. The results demonstrated the significance of the length of the optical fiber gauge on the amount of strain transferred to its core by conducting an experimental validation with both coated and bare fibers. Li et al. [5] proposed a new approach to build the mechanical model of fiber sensor by considering the protective layer 
of the fiber optic sensor as an ideal elastic-plastic material. Improved calibration formulas were presented for both compressive and tensile uniaxial strain fields and experimentally validations were conducted. Li et al. [6] modified the original assumptions in the previously established analytical model, and derived the average strain transfer rate formula. The predicted values were compared with actual test results, and they obtained the critical bond length of an optical fiber sensor for adequate sensing, and developed the equation for strain transferring in a multilayered structure. Zhou et al. [7] analyzed the strain transfer errors of their developed embedded FBG packaged strain sensors and the possible influencing parameters, and subsequently proposed correction factors for an accurate experimental strain measurement. Li et al. [8] derived the mathematical relationship between the strain from the embedded FBG sensors and that in the host material under non-axial stress. They concluded that the strain transfer models for FBG sensors subjected to axial and non-axial stress fields are very different. Sun et al. [9] used finite element analysis to evaluate which of the analytical formulas previously presented were best suited for estimating the strain transfer from the host material to the fiber Bragg grating sensors. Zhou et al. [10] developed a finite element model of an embedded FBG sensor to analyze the axial strain distribution in each layer of the material, as well as the influence of the bonding length, thickness and Young's modulus of the adhesive on the strain transfer. The numerical results were compared with experimental tensile tests on aluminum specimens. Additionally, the parameters controlling the strain transfer efficiency were also investigated.

In engineering applications, varying environmental temperature leads to a volume change in materials. Thermal effect is an important parameter, which could affect the structural 
deformations. Due to the difference in the physical properties between the optical fiber, protective layer, adhesive layer and the host structure, the volume change caused by temperature variations is significantly different in each component, which leads to thermal stress transfer between different components, resulting in inaccurate strain measurements. In order to achieve a more accurate strain measurement for the host structure, it is necessary to properly understand the strain transfer mechanism and consider the varying temperature effect.

\section{Derivation of Theoretical Formulas}

\section{The basic assumptions}

The following assumptions are adopted in this study:

(1) The bonding conditions are ideal between the fiber core and interlayer, and between interlayer and matrix, assuming there is no relative slip.

(2) The deformation of optical fiber is transferred from the shear deformation between the host material and the interlayer. The optical fiber and the inter-layer are not directly subjected to external forces, which means that only the optical fiber tip is affected by temperature effect.

(3) All the components including the optical fiber, interlayer and host material are assumed to be linear elastic before and after deformation.

(4) Only axial displacements in optical fiber and interlayer can occur and there is no radial deformation.

\section{Theoretical Derivation}

The simplified theoretical model of an embedded FBG sensor in a host structure is shown in

Fig.1, where $X-Y-Z$ is the global coordinate system; $x-y-z$ is the local coordinate system of the 
optical fiber and the interlayer; $r_{a}, r_{g}$ and $r_{m}$ represent the radius of the host material, the optical fiber and the interlayer, respectively; and $\alpha$ is the angle between the optical fiber and the direction of the principal force in the host structure.

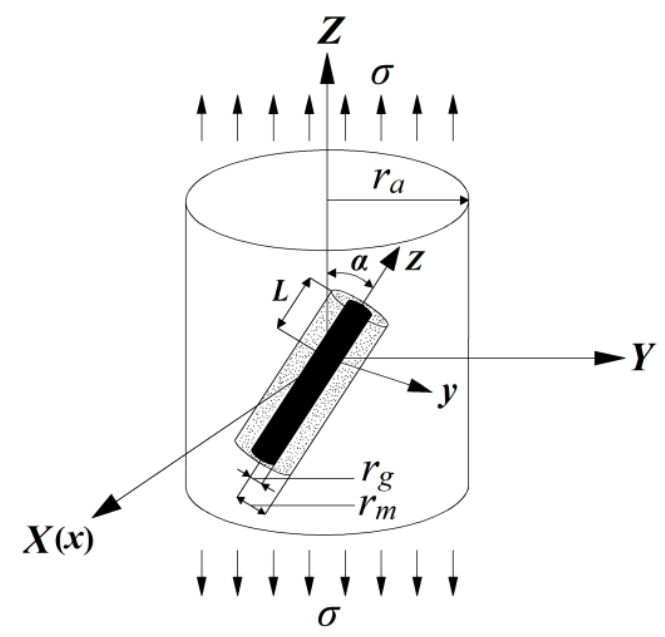

FIG. 1. Optical fiber sensor embedded in the host material (non-axial stress for FBG sensor).

When FBG sensor is embedded into the structure and subjected to the structural deformation, the stresses of an infinitesimal body are obtained based on the above-mentioned assumptions and shear-lag theory, as shown in Fig. 2. $\mathrm{d} z$ represents the length of the chosen micro-segment of the fiber, $\sigma_{g}, \sigma_{c}$, and $\sigma_{m}$ represent the normal stresses of the fiber, interlayer and transversal section of the host material respectively. $\tau_{g c}\left(z, r_{g}\right)$ denotes the shear stress in the interface between the fiber and interlayer, $\tau_{c r}(z, r)$ denotes the shear stress on the interlayer with an external diameter $r$, and $\sigma$ represents the normal stress in the $Z$ direction of the host material.
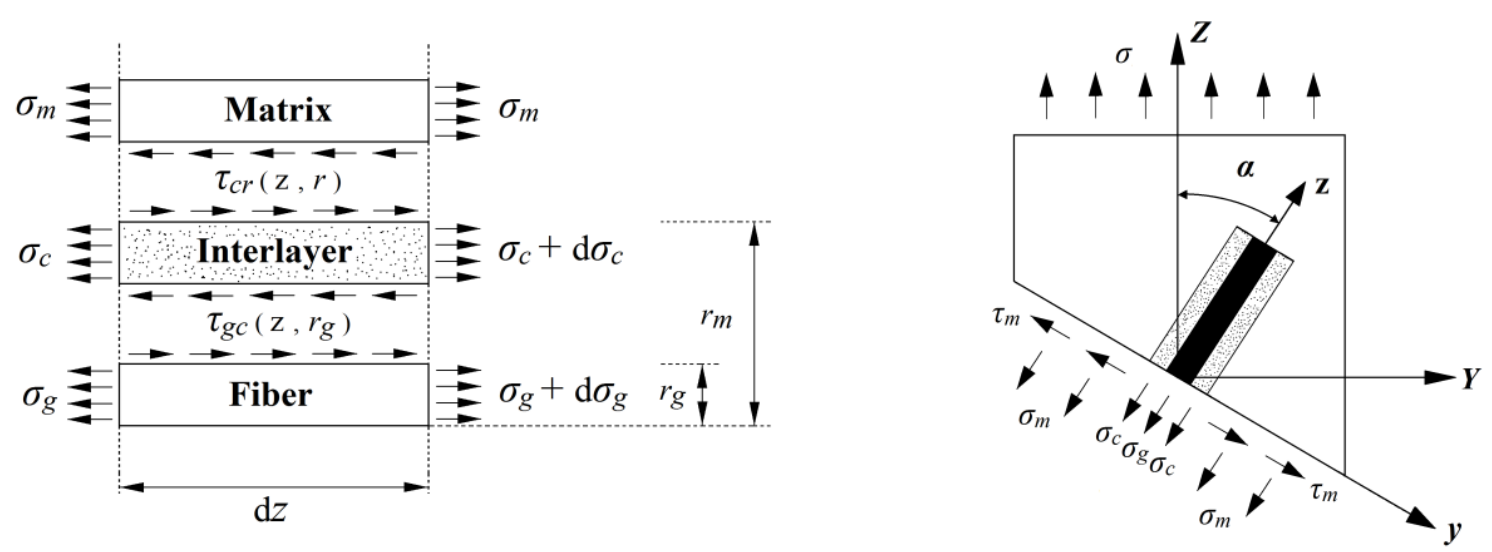
FIG. 2. Stresses on a chosen micro-element in a symmetrical coordinate system.
FIG. 3. Analysis of overall stress in global cylindrical coordinate system.

The stresses on the micro-element of the fiber are shown in Fig. 2, in which radial deformation of the fiber is neglected. Based on equilibriums, the following equation can be obtained

$$
\frac{\mathrm{d} \sigma_{g}}{\mathrm{~d} z}=-\frac{2 \tau_{g c_{\mathrm{c}}\left(r, r_{g}\right.}}{r_{g}}
$$

Similarly, the equilibrium equation for the interlayer can be written as

$$
\tau_{c r}(z, r)=\frac{r_{g}}{r} \tau_{g c}\left(z, r_{g}\right)-\frac{r^{2}-r_{g}^{2}}{2 r} \frac{\mathrm{d} \sigma_{c}}{\mathrm{~d} z}
$$

Based on the overall equilibrium along $z$ direction of the micro-element as shown in Fig. 3, the following formula can be obtained

$$
r_{g}^{2} \sigma_{g}+\left(r_{m}^{2}-r\right)_{g}^{2} \sigma+\left(\frac{r_{a}^{2}}{\mathrm{co}^{2} \mathrm{~s} \alpha}-r^{2}\right)_{m} \sigma={ }_{m} r^{2} \sigma_{a} \mathrm{~s}
$$

The deformation compatibility equation, which defines the relative displacement between the fiber and the host material, is determined from the shear strain $\gamma_{c}$ in the interlayer and can be expressed as follows

$$
u_{m}-u_{g}=\int_{r_{g}}^{r_{m}} \gamma(\eta) \mathrm{d}
$$

where $u_{m}$ and $u_{g}$ represent the deformation of the outer boundary of the interlayer and the fiber core along the $z$ direction, respectively; and $\gamma_{c}$ is the shear strain of the interlayer.

Differentiating both sides of Eq. (4) with respect to $z$, it has

$$
\frac{\mathrm{d} u_{m}}{\mathrm{~d} z}-\frac{\mathrm{d} u_{g}}{\mathrm{~d}}=\varepsilon_{m}-\varepsilon_{g}=\int_{r_{g}}^{r_{m}} \frac{\Phi_{c} x}{\mathrm{~d}} \mathrm{~d} r
$$

where $\varepsilon_{\mathrm{m}}$ and $\varepsilon_{g}$ are the strains in the host material and the fiber along the $z$ direction, respectively. They can be obtained, with considering the temperature variation effect, as

$$
\varepsilon_{m}=\frac{\sigma_{m}}{E_{m}}+\alpha_{m} \Delta T \quad \varepsilon=\frac{\sigma_{g}}{E_{g}}+\alpha \Delta \Delta_{g} T
$$




$$
\tau_{c r}=G_{c} \gamma_{c}(r)
$$

Substituting Eq. (6) into Eq. (5), the following equation is obtained

$$
\left(\frac{\sigma_{m}}{E_{m}}+\alpha_{m} \cdot \Delta T\right)-\left(\frac{\sigma_{g}}{E_{g}}+\alpha_{g} \cdot \Delta T\right)=\frac{1}{G_{c}} \int_{r_{g}}^{r_{m}} \frac{\mathrm{d} \tau_{c}(r)}{\mathrm{d} z} \mathrm{~d} r
$$

where $E_{m}$ and $E_{g}$ represent the Young's modulus of the host material and the fiber, respectively; $G_{c}$ is the shear modulus of interlayer; $\alpha_{m}$ and $\alpha_{g}$ denote respectively the thermal expansion coefficients of the host material and fiber; and $\Delta T$ denotes the temperature variation.

Substituting Eqs. (1)- (3) into Eq.(7), the following equation can be obtained

$$
\begin{aligned}
& \left\{\frac{1}{E_{m}}\left[r_{a}^{2} \sigma \cos \alpha-r_{g}{ }^{2} \sigma_{g}-\left(r_{m}{ }^{2}-r_{g}\right)^{2} \sigma_{c}\right] \cdot \frac{1}{r_{a}^{2} / \cos ^{2} \alpha-r_{m}}{ }^{2}+\alpha_{m} \cdot \Delta T\right\}-\varepsilon_{g} \\
& =-\frac{1}{G_{c}} \int_{r_{s}}^{r_{m}} \frac{\mathrm{d}}{\mathrm{d} z}\left[\frac{r^{2}-r_{g}{ }^{2}}{2 r} \frac{\mathrm{d} \sigma_{c}}{\mathrm{~d} z}+\frac{r_{g}}{2 r} \frac{\mathrm{d} \sigma_{g}}{\mathrm{~d} z}\right] \mathrm{d} r
\end{aligned}
$$

Integrating the right side of Eq. (8), it has

$$
\begin{aligned}
& \left\{\frac{1}{E_{m}}\left[r_{a}^{2} \sigma \cos \alpha-r_{g}^{2} \sigma_{g}-\left(r_{m}{ }^{2}-r_{g}\right)^{2} \sigma_{c}\right] \cdot \frac{1}{r_{a}^{2} / \cos ^{2} \alpha-r_{m}}{ }^{2}+\alpha_{m} \cdot \Delta T\right\}-\varepsilon_{g} \\
& =-\frac{1}{G_{c}} \frac{\mathrm{d}}{\mathrm{d} z}\left\{\frac{1}{2}\left[\frac{1}{2}\left(r_{m}^{2}-r_{g}{ }^{2}\right)-r_{g} \ln \left(\frac{r_{m}}{r_{g}}\right)\right] \frac{\mathrm{d} \sigma_{c}}{\mathrm{~d} z}+\frac{1}{2} r_{g} \ln \left(\frac{r_{m}}{r_{g}}\right) \frac{\mathrm{d} \sigma_{g}}{\mathrm{~d} z}\right\}
\end{aligned}
$$

Because of the simultaneous deformations of FBG sensor, interlayer and host material, the strains in these three components are almost the same. Since Young's moduli of the fiber and host material are usually at least ten times greater than that of the interlayer, the following equation can be obtained

$$
\begin{gathered}
\frac{E_{\mathrm{c}}}{E_{m}} \cong \frac{E_{\mathrm{c}}}{E_{g}} \cong 0 \\
\because \sigma=E_{m}\left(\varepsilon-\alpha_{m,} \Delta \mathrm{T}\right) \quad \sigma_{g}=E \&{ }_{g} \alpha \Delta \mathrm{T}_{g} \\
\therefore \frac{\sigma_{c}}{E_{m}} \cong 0 \quad \frac{\sigma_{c}}{E_{g}} \cong 0
\end{gathered}
$$

Substituting Eq. (10) into Eq. (9), it has 


$$
\begin{aligned}
& {\left[r_{a}^{2} \cos \alpha\left(\varepsilon-\alpha_{m} \Delta T\right)-\frac{E_{g}}{E_{m}} r_{g}^{2}\left(\varepsilon_{g}-\alpha_{g} \Delta T\right)\right] \cdot \frac{\cos ^{2} \alpha}{r_{a}^{2}-r_{m}^{2} \cos ^{2} \alpha}+\alpha_{m} \cdot \Delta T-\varepsilon_{g}} \\
& =-\frac{E_{g}}{G_{c}} \cdot \frac{1}{2} r_{g}^{2} \ln \left(\frac{r_{m}}{r_{g}}\right) \frac{\mathrm{d}^{2} \varepsilon_{g}}{\mathrm{~d} z^{2}}
\end{aligned}
$$

Eq. (11) can be sorted out as follows

$$
\begin{aligned}
& \frac{E_{g}}{G_{c}} \cdot \frac{1}{2} r_{g}^{2} \ln \left(\frac{r_{m}}{r_{g}}\right) \frac{\mathrm{d}^{2} \varepsilon_{g}}{\mathrm{~d} z^{2}}-\left[\frac{\left(E_{g} / E_{m}\right) r_{g}^{2} \cos ^{2} \alpha}{r_{a}^{2}-r_{m}^{2} \cos ^{2} \alpha}+1\right] \varepsilon_{g} \\
& =-\left(\frac{r_{a}^{2} \cos \alpha}{r_{a}^{2}-r_{m}^{2} \cos ^{2} \alpha}\right) \varepsilon+\left(\frac{r_{a}^{2} \cos ^{2} \alpha}{r_{a}^{2}-r_{m}^{2} \cos ^{2} \alpha}-1\right) \alpha_{m} \Delta T-\frac{\left(E_{g} / E_{m}\right) r_{g}^{2} \cos ^{2} \alpha}{r_{a}^{2}-r_{m}^{2} \cos ^{2} \alpha} \cdot \alpha_{g} \Delta T
\end{aligned}
$$

Defining the following two coefficients $\eta$ and $\xi$,

$$
\begin{gathered}
\eta^{2}=\left[\frac{\left(E_{g} / E_{m}\right) r_{g}^{2} \cos ^{2} \alpha}{r_{a}^{2}-r_{m}^{2} \cos ^{2} \alpha}+1\right] /\left[\frac{E_{g}}{G_{c}} \cdot \frac{1}{2} r_{g}^{2} \ln \left(\frac{r_{m}}{r_{g}}\right)\right] \\
\xi^{2}=\left[\frac{r_{a}^{2} \cos ^{2} \alpha}{r_{a}^{2}-r_{m}^{2} \cos ^{2} \alpha}\right] /\left[\frac{E_{g}}{G_{c}} \cdot \frac{1}{2} r_{g}^{2} \ln \left(\frac{r_{m}}{r_{g}}\right)\right]
\end{gathered}
$$

Eq. (12) can be expressed as

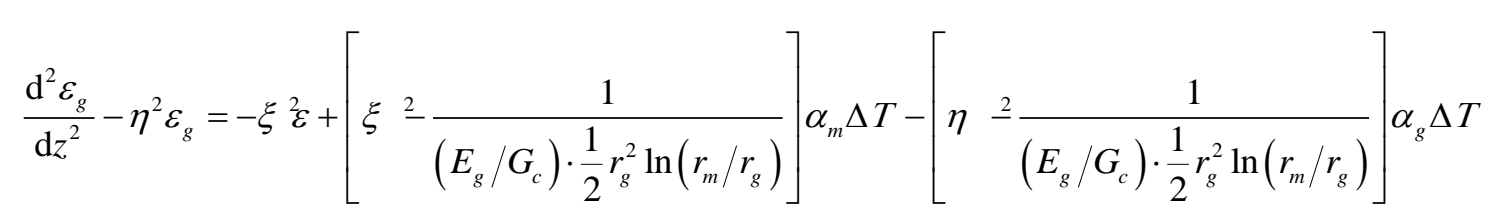

Eq. (15) is the differential equation of the strain relationship between the fiber and host material for the embedded FBG sensor when influenced by non-axial stress and temperature variation, in which coefficients $\eta$ and $\xi$ reflect the influences of radius and Young's modules of fiber and interlayer. The solution of Eq. (15) can be obtained as

$$
\begin{aligned}
& \varepsilon_{g}(z)=\mathrm{A} \sinh (\eta z)+B \cosh (\eta z)+\frac{\xi^{2}}{\eta^{2}} \varepsilon-\left[\frac{\xi^{2}}{\eta^{2}}-\frac{1}{\eta^{2}\left(E_{g} / G_{c}\right) \cdot \frac{1}{2} r_{g}^{2} \ln \left(r_{m} / r_{g}\right)}\right] \alpha_{m} \Delta T \\
& +\left[1-\frac{1}{\eta^{2}\left(E_{g} / G_{c}\right) \cdot \frac{1}{2} r_{g}^{2} \ln \left(r_{m} / r_{g}\right)}\right] \alpha_{g} \Delta T
\end{aligned}
$$


The constants $A$ and $B$ are determined by the boundary conditions. Based on the assumptions in Section 2.1, the optical fiber and the interlayer are not directly subject to external forces, that is, there is only thermal strain at the optical fiber tip, thus the boundary conditions are represented as

$$
\varepsilon_{g}(L)=\varepsilon_{g}(-L)=\alpha_{g} \Delta T
$$

where $L$ is the half length of the fiber.

Substituting Eq. (17) into Eq. (16), $A$ and $B$ can be obtained as follows,

$$
\begin{gathered}
A=0 \\
B=\frac{1}{\cosh (\eta L)}\left[-\frac{\xi^{2}}{\eta^{2}} \varepsilon+\frac{\xi^{2}}{\eta^{2}} \alpha_{m} \Delta T-\frac{\alpha_{m} \Delta T}{\eta^{2}\left(E_{g} / G_{c}\right) \cdot \frac{1}{2} r_{g}^{2} \ln \left(r_{m} / r_{g}\right)}+\frac{1}{\eta^{2}\left(E_{g} / G_{c}\right) \cdot \frac{1}{2} r_{g}^{2} \ln \left(r_{m} / r_{g}\right)} \alpha_{g} \Delta T\right]
\end{gathered}
$$

Therefore the axial strain of the fiber affected by the non-axial stress and temperature variation is

$$
\begin{aligned}
\varepsilon_{g}(z) & =\left[1-\frac{\cosh (\eta z)}{\cosh (\eta L)}\right] \frac{\xi^{2}}{\eta^{2}} \varepsilon-\left[1-\frac{\cosh (\eta z)}{\cosh (\eta L)}\right] \cdot\left[\frac{\xi^{2}}{\eta^{2}}-\frac{1}{\eta^{2}\left(E_{g} / G_{c}\right) \cdot \frac{1}{2} r_{g}^{2} \ln \left(r_{m} / r_{g}\right)}\right] \alpha_{m} \Delta T \\
& +\left[1+\frac{\cosh (\eta z)-\cosh (\eta L)}{\cosh (\eta L) \cdot \eta^{2}\left(E_{g} / G_{c}\right) \cdot \frac{1}{2} r_{g}^{2} \ln \left(r_{m} / r_{g}\right)}\right] \alpha_{g} \Delta T
\end{aligned}
$$

A FBG sensor consists of a fiber core, the cladding and coating layers. When applied to general structural health monitoring exercises, the outer radius of the middle layer is much smaller than the radius of the host structure, that is , $r_{m} \ll r_{a}$.

The following equations can be derived based on the formulas $r_{m} \ll r_{a}$ and $G_{\mathrm{c}}=\frac{E_{c}}{2\left(1+v_{c}\right)}$

$$
\eta^{2}=\frac{1}{\left(1+v_{c}\right)\left(E_{g} / E_{c}\right) r_{g}^{2} \ln \left(r_{m} / r_{g}\right)}
$$




$$
\xi^{2}=\frac{\cos ^{2} \alpha}{\left.\left(1+v_{c}\right) E_{g} / E_{c}^{2}\right)_{g} 1 \ln _{m}\left(r_{g}\right)}=\eta^{2} \cos ^{2} c
$$

where $v_{c}$ is Poisson's ratio of the interlayer.

Substituting Eqs. (20) and (21) into Eq.(19), it has

$$
\varepsilon_{g}(z)=\cos ^{2} \alpha\left[1-\frac{\cosh (\eta z)}{\cosh (\eta L)}\right] \varepsilon+\left[1-\frac{\cosh (\eta z)}{\cosh (\eta L)}\right] \cdot\left(1-\cos ^{2} \alpha\right) \alpha_{m} \Delta T+\frac{\cosh (\eta z)}{\cosh (\eta L)} \cdot \alpha_{g} \Delta T
$$

Eq. (22) defines the strain transfer relationship between the FBG sensor and the host structure when subjected to the non-axial stress and temperature variation. When both the angle and temperature variation are equal to zeros, Eq. (22) can be simplified as the strain transfer formula given by $\mathrm{Li}$ et al. [6]. When the temperature variation is not taken into consideration, Eq. (22) is degraded as the strain transfer formula derived by Li and Zhou [8]. As can be seen from the above formulas, when ignoring the temperature, under non-axial stress and temperature variation, the strain relationship between FBG sensor and structure is different from that under axial stress. It should be noted that although Eq. (22) is the strain transfer relationship derived from the FBG sensor, it is also suitable for other fiber sensors which measure strains depending on the deformation of the fiber core.

Strain transfer from the fiber bonding position can be defined as the ratio of FBG sensor measurement strain to the actual strain of the position. It can be expressed as

$$
\mu(z)=\frac{\varepsilon_{g}(z)}{\varepsilon}
$$

\section{Finite Element Method (FEM)}

The results from the proposed theoretical method are verified by the FEM analysis of a concrete cylinder of diameter $150 \mathrm{~mm}$ and height $150 \mathrm{~mm}$ as the host structure with the optical 
fiber embedded into the cylinder with a $10^{\circ}$ angle from the principal stress direction (as shown in Fig. 1). Finite element model is established and the meshing of internal fiber is shown in Fig.4. Eight node elements are used to generate meshes. The length of the fiber is $40 \mathrm{~mm}$. Material properties of the fiber, the interlayer and the concrete matrix are given in Table 1.

TABLE 1. Material properties.

\begin{tabular}{|c|c|c|c|}
\hline Materials parameters & Symbols & Values & Units \\
\hline Young's modulus of the fiber core & $E_{g}$ & $7.2 \times 10^{4}$ & $\mathrm{MPa}$ \\
\hline Young's modulus of the interlayer & $E_{c}$ & 2.55 & $\mathrm{MPa}$ \\
\hline Young's modulus of the matrix concrete & $E_{m}$ & $2.2 \times 10^{4}$ & $\mathrm{MPa}$ \\
\hline Poisson's ratio of the fiber core & $v_{g}$ & 0.17 & - \\
\hline Poisson's ratio of the interlayer & $v_{c}$ & 0.48 & - \\
\hline Poisson's ratio of the matrix concrete & $v_{m}$ & 0.18 & - \\
\hline Radius of the fiber core & $r_{g}$ & 62.5 & $\mu \mathrm{m}$ \\
\hline Radius of the outer boundary of the interlayer & $r_{m}$ & 102.5 & $\mu \mathrm{m}$ \\
\hline Coefficient of thermal expansion of the fiber core & $\alpha_{\mathrm{g}}$ & $0.55 \times 10^{-6}$ & - \\
\hline Coefficient of thermal expansion of the interlayer & $\alpha_{c}$ & $45 \times 10^{-6}$ & - \\
\hline Coefficient of thermal expansion of the matrix concrete & $\alpha_{\mathrm{m}}$ & $10 \times 10^{-6}$ & - \\
\hline
\end{tabular}

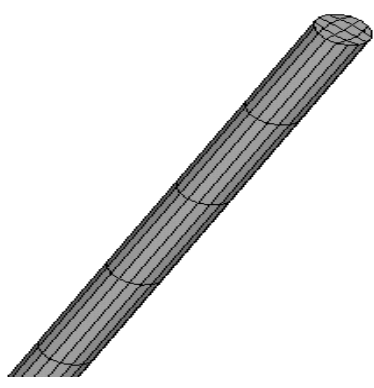

(a) Local mesh of the optical fiber

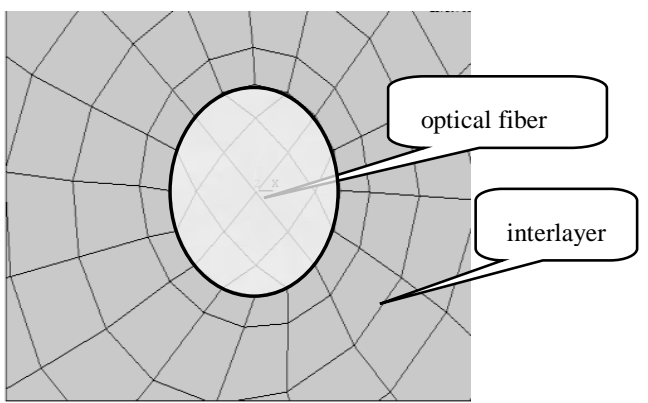

(b) Mesh interface between the optical fiber and interlayer

FIG. 4. Mesh of the embedded optical fiber.

The initial temperature is set as $0{ }^{\circ} \mathrm{C}$ and temperature variation is $50{ }^{\circ} \mathrm{C}$, and the applied uniform tensile stress on the concrete column is set as $2 \mathrm{MPa}$. The analytical strain distribution 
in the embedded FBG sensor under non-axial stress is obtained by the finite element analysis and shown in Fig. 5. The comparison between the strains obtained by the finite element analysis and the theoretical strain from Equation (22) is shown in Fig. 6.

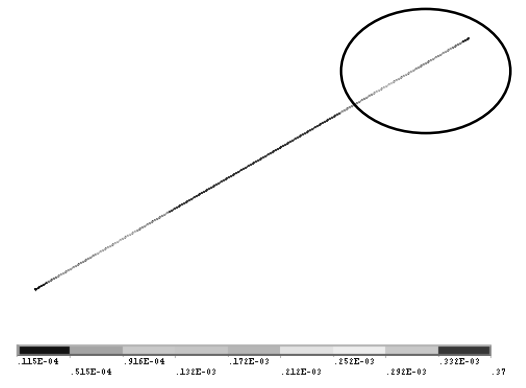

(a) The whole strain of the fiber along the $z$ direction

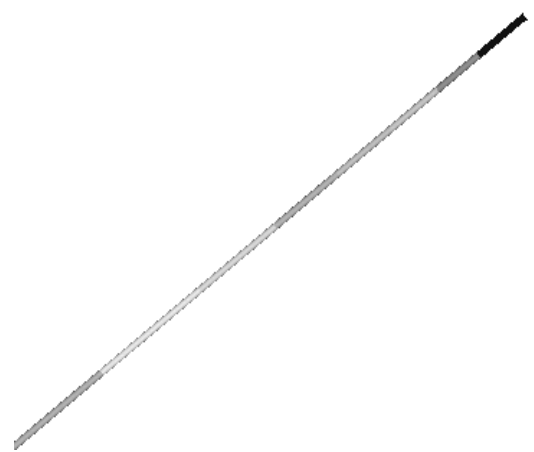

(b) The local strain of the fiber along the $z$ direction

FIG. 5. The strain of the fiber along the $z$ direction.

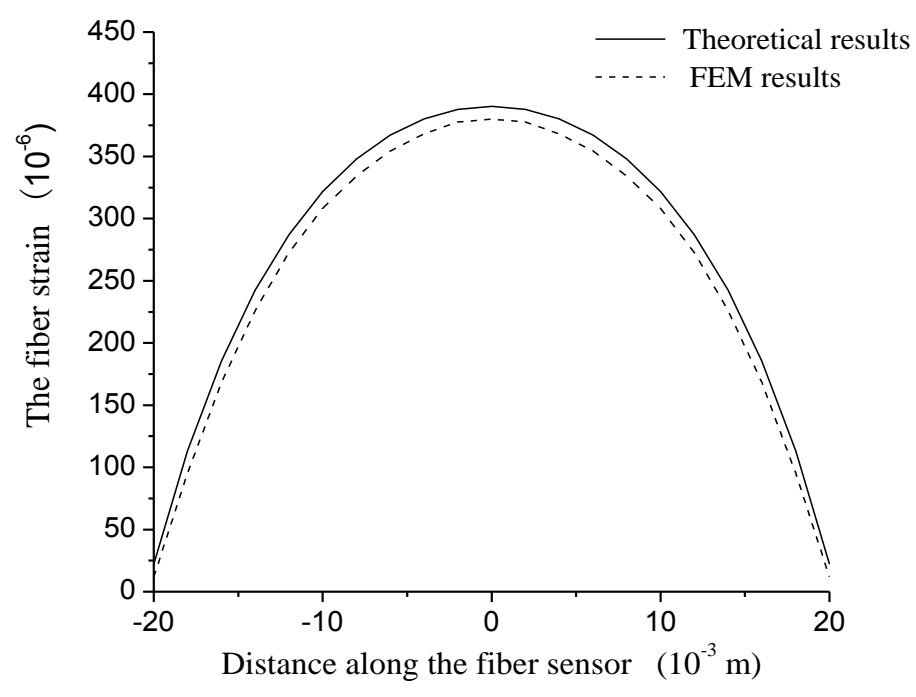

FIG. 6. Comparison between the finite element analysis result and theoretical result

As can be seen from Fig. 5, the strain in the optical fiber varies gradually. From Fig. 6, the largest strain is observed in the middle of the fiber, and the strain is decreasing gradually to zeros at the both ends. The strain obtained by the finite element method agrees well with the theoretical result by the proposed strain transfer equation with the average error less than $5 \%$. 


\section{Error Analysis}

In practical applications, the positioning of FBG sensors, function of the host material and environmental conditions may cause substantial temperature variations. The strain transfer rate may be changed and thus the measurement accuracy is affected. It is easy to understand that the temperature changes will have an effect on the measurement accuracy from FBG sensors. However, it is not clear how large a temperature change will induce unacceptable errors in the strain measurement. In this section, the effect of temperature variation on the strain measurement accuracy is investigated.

The relative error in the strain measurement can be defined as

$$
\delta=\frac{\varepsilon^{\prime}-\varepsilon}{\varepsilon}
$$

where $\varepsilon^{\prime}$ is the measured structural strain and $\varepsilon$ is the true one.

The strain measured by the FBG sensor is the average strain within the range of the whole adhesive length and can be expressed as

$$
\begin{gathered}
\bar{\varepsilon}_{g}=2 \int_{0}^{L} \frac{\varepsilon_{g}(z)}{2 L} \mathrm{~d} z \\
\bar{\varepsilon}_{g}=\cos ^{2} \alpha\left[1-\frac{\sinh (\eta L)}{\eta L \cosh (\eta L)}\right] \varepsilon+\left(1-\cos ^{2} \alpha\right)\left[1-\frac{\sinh (\eta L)}{\eta L \cosh (\eta L)}\right] \alpha_{m} \Delta T \\
+\frac{\sinh (\eta L)}{\eta L \cosh (\eta L)} \cdot \alpha_{g} \Delta T
\end{gathered}
$$

The average strain transfer rate is

$$
\bar{\mu}=\frac{\bar{\varepsilon}_{g}}{\varepsilon}
$$

Introducing Eq. (27) into Eq. (24), the following equation can be obtained

$$
\delta=\frac{\bar{\mu}-\bar{\mu}^{\prime}}{\bar{\mu}^{\prime}}
$$


where $\bar{\mu}^{\prime}$ represents the average strain transfer rate when considering the temperature variation.

Calculated from Eq. (26)-Eq. (28), errors caused by the temperature difference are shown in Fig. 7, with the same parameters in the finite element analysis of Section 3. When the temperature difference is larger than $20^{\circ} \mathrm{C}$, the relative error in the strain measurement is more than $5 \%$. If we consider a relative error less than $5 \%$ as an acceptable error limit in practical application of strain measurement by using FBG sensors, it is necessary to consider the influence of the temperature difference when it is larger than $20^{\circ} \mathrm{C}$.

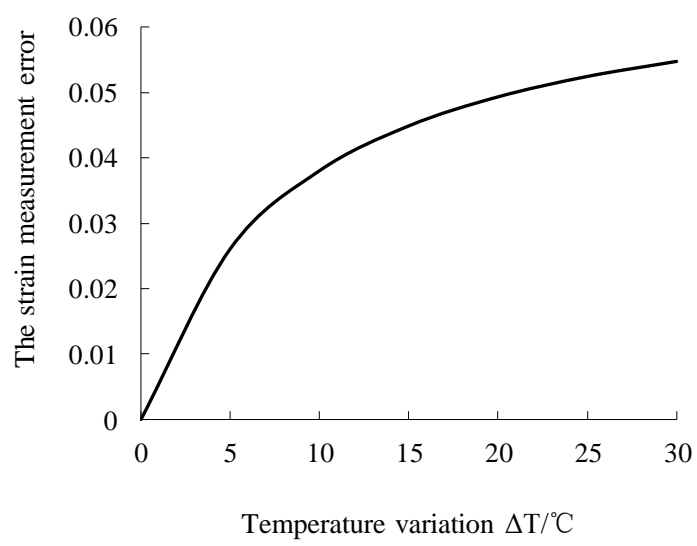

FIG. 7. Relative error of the strain measurement with the temperature variation.

In practice, the FBG sensor placement might not be exactly aligned with the desired strain measurement direction, which leads to errors in the measured strains. The influence of this scenario is studied by assuming a misalignment angle $\Delta \alpha$ between the optical fiber and the principal stress direction in the concrete column of $1^{\circ}, 2^{\circ}$ and $3^{\circ}$, respectively. The initial temperature variation is set as $\Delta T=20^{\circ} \mathrm{C}$. Fig. 8 shows the relationship between the strain measurement error and the variation of the misalignment angle with respect to the desired embedment angle of FBG sensors. Generally for these three cases with small errors in the 
placement angles, the strain measurement error becomes larger with the increase of the embedment angle. When the embedded angle of FBG sensors is greater than $60^{\circ}$, the strain measurement error increases dramatically. A slight angle deviation will lead to a huge error. In the design of laying angles of FBG sensors, the angle should be less than $60^{\circ}$.

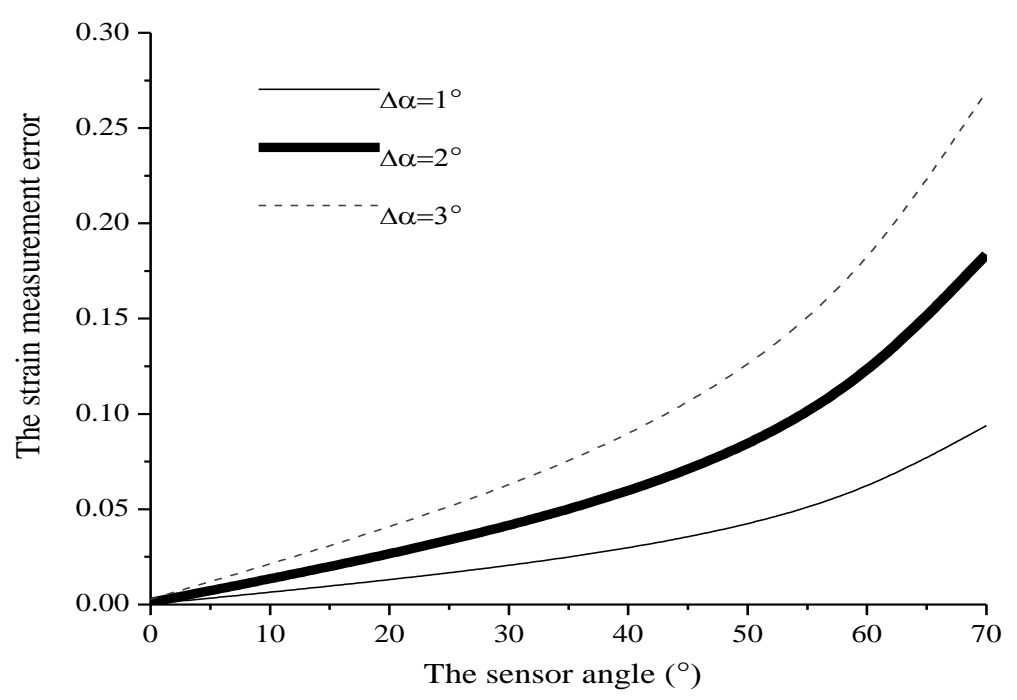

FIG. 8. Variation of the strain measurement error with the embedded angle.

\section{Influences of Temperature Difference and Embedded Angle}

With the physical properties listed in Table 1 , when $\alpha$ is defined to be equal to $10^{\circ}, \sigma$ is set as $2 \mathrm{MPa}$ and the temperature variations are selected as $0^{\circ} \mathrm{C}, 15^{\circ} \mathrm{C}, 30^{\circ} \mathrm{C}$ respectively, the strains in the fiber and the strain transfer rates along the scale distance of the FBG sensors are shown in Fig. 9 and Fig. 10, respectively. It can be observed from Fig. 9 that the bigger the temperature difference, the larger the measurement strains in the optical fiber. As can be seen from Fig. 10, when $\Delta T$ equals $15^{\circ} \mathrm{C}$ and $30^{\circ} \mathrm{C}$ respectively, the curves of the strain transfer rate are quite close to each other. The largest strain transfer rate occurs at the center of the optical fiber, and it gradually decreases to zeros at the two ends. Compared with the results in Fig. 9, the temperature difference has a less influence on the strain transfer rate under the same angle. 


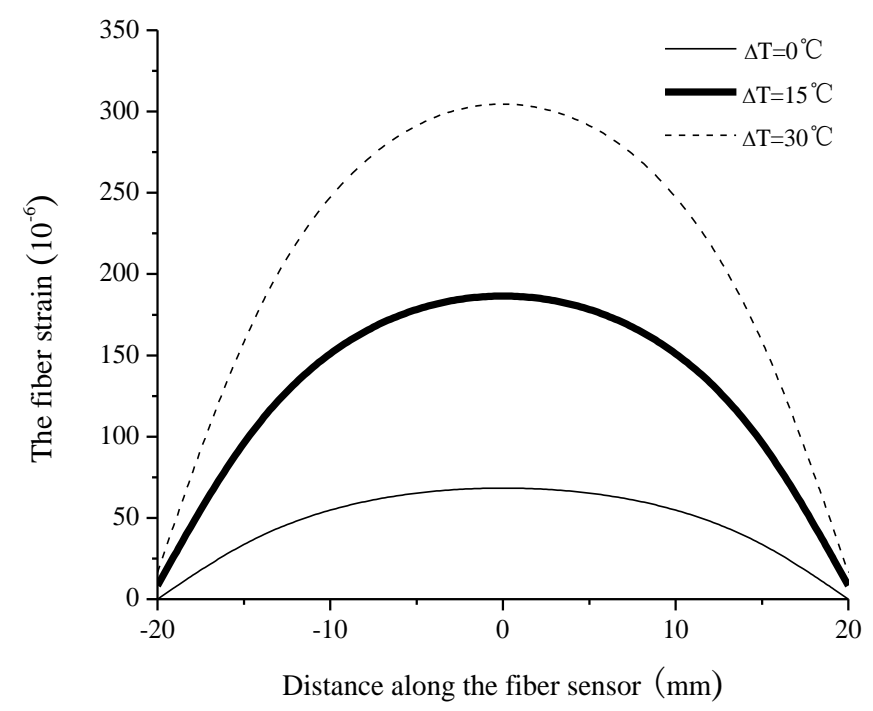

FIG. 9. Strain of the optical fiber under different temperature differences.

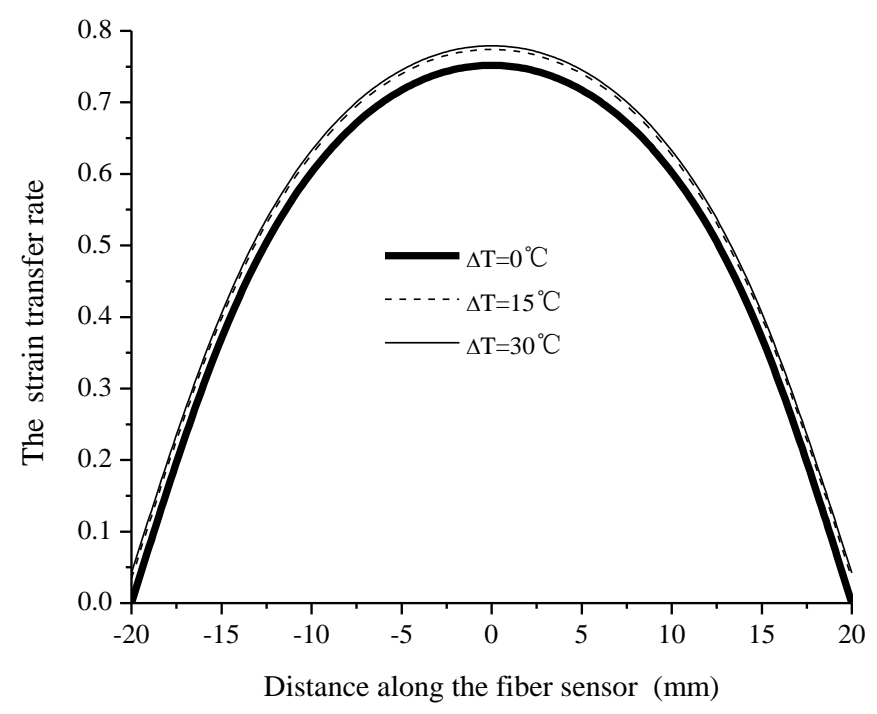

FIG. 10. Strain transfer rate of the optical fiber under different temperature differences.

When $\sigma$ is defined as $2 \mathrm{MPa}, \Delta \mathrm{T}$ as $50^{\circ} \mathrm{C}$ and $\alpha$ as $0^{\circ}, 20^{\circ}, 40^{\circ}$ respectively, the calculated strains and strain transfer rates in the fiber are shown in Fig. 11 and Fig. 12, respectively. The fiber strain decreases with the embedded angle, as well as the strain transfer rate. The maximum fiber strain and strain transfer rate are located at the middle of the fiber, and these values become smaller when approaching the two ends of the fiber. 


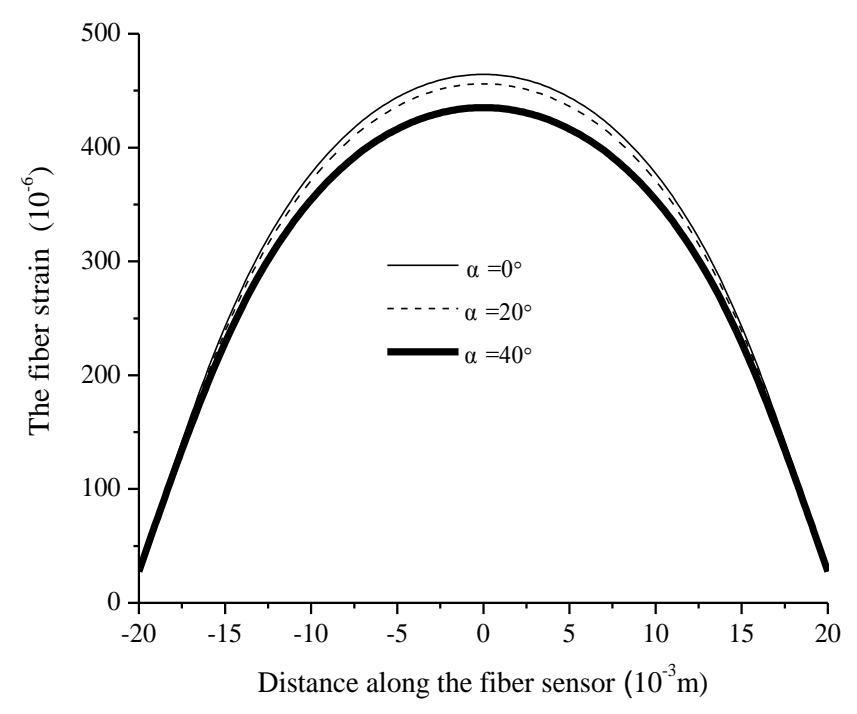

FIG. 11. Strain distribution of the optical fiber along the length under different angles.

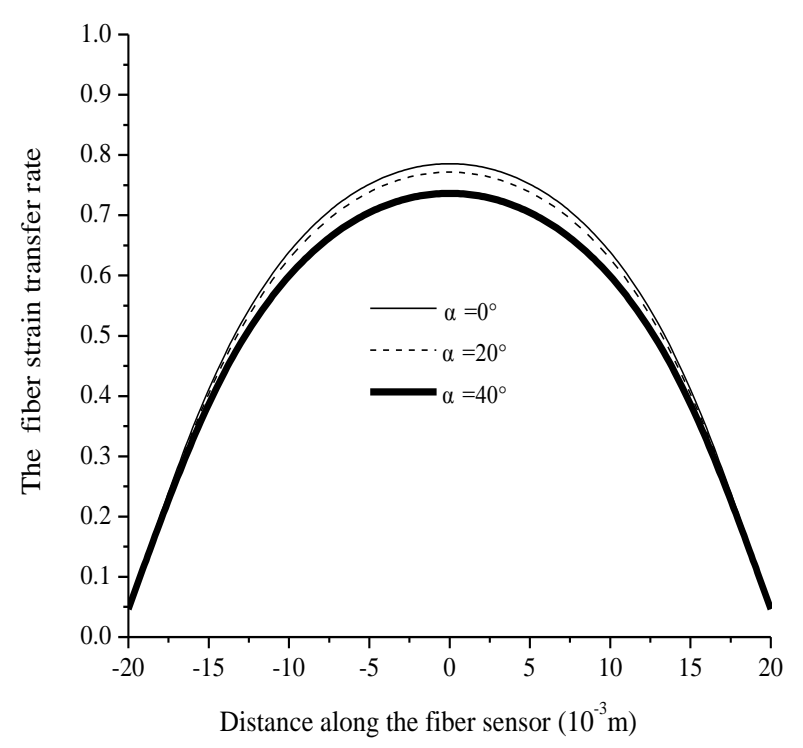

FIG. 12. Distribution of the strain transfer rate of the optical fiber along the length under different angles.

\section{Conclusion}

Based on the fundamentals of the shear-lag method and a series of appropriate assumptions for the interaction of the optical fiber and host material, the relationship of the strains between the FBG sensor and the host structure is established when the fiber is subjected to the non-axial stress and temperature effect. The accuracy and effectiveness of the proposed formulas are 
verified by numerical analysis. The strain transfer rate along the length of FBG sensors and the average strain value in the fiber are obtained. The influence of the temperature variation on the strain measurement error is investigated. Moreover, the possible errors associated with the influences of several parameters, i.e. the embedded angle and temperature deviation on the average strain transfer rate are also analyzed and quantified.

The proposed formulas can also be applied to other optical fiber sensors that depend on the deformation of the central optical fiber and sealed FBG sensors. Under the effect of both temperature and non-axial stress loading conditions, the transfer relationship between the strain measured by the optical fiber and the analytical strain from finite element analysis is obtained, and the measurement error is analyzed to provide the design and installation guidelines for FBG sensors. The strain measurement accuracy can be improved with the use of the proposed strain transfer formulas and the error analysis results.

\section{Acknowledgement}

This work has been supported by the National Natural Science Foundation of China (51178277), Australia Endeavour Research Fellowship (3815_2014), Program for New Century Excellent Talents in University (NCET-12-1013), Program for Liaoning Excellent Talents in University （LR2012018）, Liaoning BaiQianWan Talents program (2014921045) and the Shenyang City Science Foundation of China (F14-192-4-00). 


\section{References}

[1] Cox, H. L., "The elasticity and strength of paper and other fibrous materials," Britain Journal of Applied Physics, Vol. 3(3), 1952, pp.72-79.

[2] Nanni, A., Yang, C. C., Pan, K., Wang, J. S., and Michael, R. R., "Fiber-optic sensors for concrete strain/stress measurement,” ACI Materials Journal, Vol.88 (3), 1991, pp. 257-264.

[3] Pak, Y. E., "Longitudinal shear transfer in fiber optic sensors", Smart Mater and Structures, Vol. 1, 1992, pp. 57-62.

[4] Ansari, F., Yuan, L. B., "Mechanics of bond and interface shear transfer in optical fiber sensors," Journal of Engineering Mechanics, Vol.124 (4), 1998, pp.385-394.

[5] Li, G., Li, Q. B., Zhang, W. C., "Ideal elasto-plastic model for internal strain measurement by fiber optic sensors ," Journal of Tsinghua University, Vol.41(11), 2001, pp. 102-105. (in Chinese)

[6] Li, D. S., Li, H. N., Ren, L., and Song, G. B., "Strain transferring analysis of fiber Bragg grating sensors," Optical Engineering, Vol. 45(2), 2005, pp. 024401.

[7] Zhou, Z., Li, J. L., and OU, J. P., "Interface strain transfer mechanism and error modification of embedded FBG strain sensors," Journal of Harbin Institute of Technology, Vol. 38(1), 2006, pp.49-55. (in Chinese)

[8] Li, H. N., Zhou, G. D., Ren, L., Li, D. S., "Strain transfer analysis of embedded fiber Bragg grating sensor under nonaxial stress," Optical Engineering, Vol. 46 (5), 2007, pp.054402.

[9] Sun, L., Liang, D. Z., Zhang, H., "Comparison of several strain transfer theory calculation methods of the embedded FBG strain sensors," International Conference on Smart Materials and Nanotechnology in Engineering, Harbin, 2007, pp. 64233Z. 
[10] Zhou, J. H., Zhou, Z. D., and Zhang, D. S., "Study on strain transfer characteristics of fiber Bragg grating sensors," Journal of Intelligent Material Systems and Structures, Vol. 21(11), 2010, pp. $1117-1122$. 